\title{
WAVE PROCESSES IN NANOSTRUCTURES \\ FORMED BY NANOTUBE ARRAYS OR NANOSIZE CRYSTALS
}

\author{
V. A. Eremeyev ${ }^{1,2}$, E. A. Ivanova ${ }^{3}$, and D. A. Indeitsev ${ }^{4}$
}

Basic parameters of wave processes in structures formed by arrays of parallel nanosize crystals or nanotubes grown in the direction normal to the substrate are obtained. This problem is considered in modeling the behavior of nanoelectromechanical systems, for instance, sensors that utilize such structures. The parameters obtained can also be used to determine the effective elastic characteristics of nanoobjects forming this structure.

Key words: thin-walled structures, oscillations of elastic bodies, nanostructures.

\section{INTRODUCTION}

Application of advanced nanotechnologies allows obtaining ordered arrays formed by almost identical nanocrystals or nanotubes parallel to each other and grown in the direction normal to the substrate [1]. The interest in these structures is caused, in particular, by the development of various nanoelectromechanical systems, such as sensors, ultra violet lasers, photodetectors, etc. (see, e.g., [2-6]).

The properties of nanoobjects are substantially different from those of similar macroscopic materials $[1,7-10]$. Therefore, it seems reasonable to develop models of such nanostructures to estimate their mechanical properties. One of the most effective methods of determining the elasticity moduli is based on measuring the eigenfrequencies of the examined object. Application of this method to nanoobjects involves problems associated with difficulties in measuring their eigenfrequencies. At the same time, measuring the eigenfrequencies of the system consisting of a microsubstrate and a regular structure of identical nanoobjects is fairly realistic. A method of determining the eigenfrequencies of some nanostructures (nanotubes and nanocrystals) was proposed in [11, 12]. This method is based on measuring the eigenfrequencies of a system consisting of a vertically aligned array of identical nanotubes or nanocrystals grown on a substrate and arranged normal to the latter. Examples of nanocrystal arrays studied in $[11,12]$ are semiconductor micro- and nanocrystals of zinc oxide (Fig. 1) obtained by different methods, in particular, by means of pulsed laser deposition [13-15]. The interest in ZnO nanocrystals is caused, in particular, by the fact that they are used in ultra violet (UV) nanolasers, highly sensitive UV photodetectors, optical switches, electron emitters, solar arrays with nanostructured $\mathrm{ZnO}$ electrodes, and piezonanomechanical devices $[2,6]$.

It was demonstrated $[11,12]$ that the range of eigenfrequencies of the system consisting of the array of nanotubes (nanocrystals) and the substrate is divided into two parts. One part of the spectrum corresponds to the range of eigenfrequencies of the nanoobjects. The substrate remains almost motionless during oscillations of the nanoobjects with these frequencies. The other part of the spectrum of the system is the range of eigenfrequencies close to the eigenfrequencies of the substrate without the nanoobjects. At such frequencies, the amplitude of

\footnotetext{
1Southern Scientific Center, Russian Academy of Sciences, Rostov-on-Don 344006; eremeyev.victor@gmail.com. ${ }^{2}$ Southern Federal University, Rostov-on-Don 344006; 3Saint-Petersburg State Polytechnical University, St. Petersburg 195251; elenaivanova239@post.ru. ${ }^{4}$ Institute of Problems of Mechanical Engineering, Russian Academy of Sciences, St. Petersburg 199178; ind@director.ipme.ru. Translated from Prikladnaya Mekhanika i Tekhnicheskaya Fizika, Vol. 51, No. 4, pp. 143-154, July-August, 2010. Original article submitted November 9, 2009.
} 


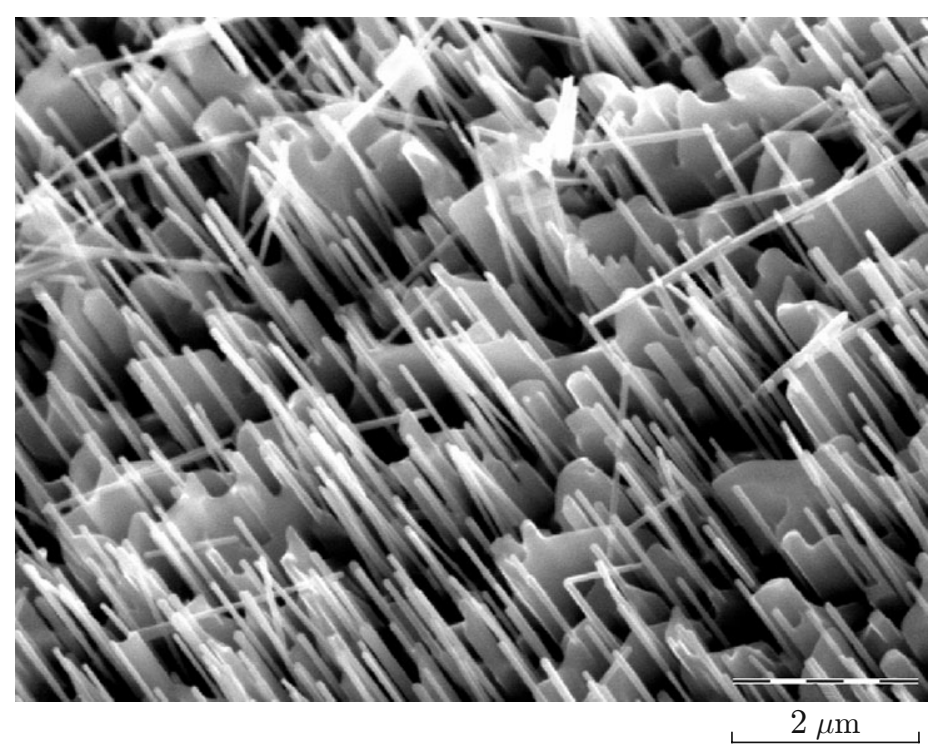

Fig. 1. Array of zinc oxide nanocrystals; the photograph was taken by K. V. Dvadnenko (Institute of Arid Zones of the Southern Scientific Center of the Russian Academy of Sciences); the sample was kindly presented by E. M. Kaidashev (Southern Federal University).

oscillations of the nanoobjects is substantially smaller than the amplitude of substrate oscillations. Thus, based on the known spectrum of the array-substrate system and the known substrate spectrum, it is possible to determine the eigenfrequencies of one nanoobject. The essence of the method is as follows. It is necessary to measure several first eigenfrequencies of the system consisting of the array of nanotubes (nanocrystals) and the substrate and several first eigenfrequencies of the same substrate without the nanoobjects. A comparison of these two spectra allows identifying frequencies in the spectrum of the system, which do not have counterparts among the frequencies in the substrate spectrum. The thus-identified frequencies are the frequencies of the nanoobjects. The most important constraint of the method proposed in $[11,12]$ is the frequency range of the measurement tools. This method is effective if the first eigenfrequencies of the nanoobjects are commensurable with the first eigenfrequencies of the substrate. If the nanoobject eigenfrequencies are too high, they cannot be detected.

A method of determining bending stiffness of nanoobjects is proposed below. This method is effective even if the nanoobject eigenfrequencies are outside the frequency range of the measurement instruments. The method is based on measuring the eigenfrequencies of a system consisting of a substrate and a regular structure of vertically aligned nanoobjects. The essence of the method is as follows. The substrate is a microobject; therefore, determining its mechanical characteristics does not involve principal difficulties. The substrate is fairly thin, and its dynamics is accurately described by the classical theory of plates. As is shown below, the dynamics of the system consisting of the substrate and a regular system of nanoobjects is described by a differential equation for transverse bending of the substrate, which has the same structure as the equation of oscillations of shear-deformable plates. It is easy to find the relation between the mechanical characteristics of nanoobjects and the effective shear stiffness. Thus, by measuring the system eigenfrequencies and knowing all mechanical characteristics of the substrate, one can determine the effective shear stiffness. If the nanoobjects are identical and are arranged regularly, then the bending stiffness of one nanoobject can be determined if the effective shear stiffness is available. It is known that the shear stiffness of plates and shells is not uniquely determined. The shear stiffness value depends (though not too much) on test conditions. To a much larger extent, the shear stiffness of the plate depends on the plate structure. Therefore, we consider two formulations of the problem to obtain more reliable results. In the first problem, the lower ends of the nanoobjects are clamped on the substrate, and the upper ends are free (Fig. 2). In the second problem, the lower ends of the nanoobjects are clamped on one substrate, and the upper ends are clamped on another identical substrate (Fig. 3). The nanoobjects are assumed to interact with each other. Such a structure can be obtained, for instance, by filling an array of nanocrystals with a polymer material, processing of the surface, and applying a new layer onto the surface. The second problem is also of interest in studying deformations of nanocomposite plates. 


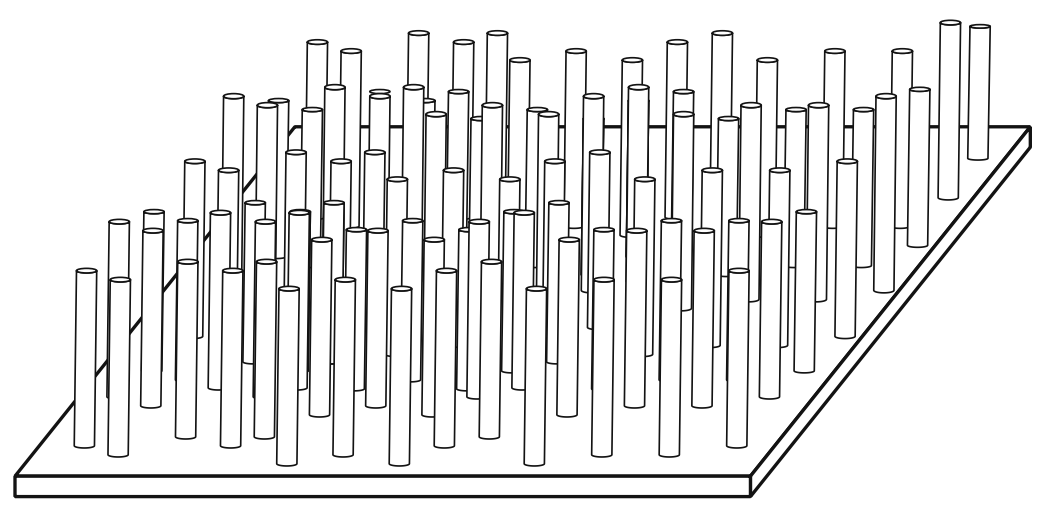

Fig. 2. Array of nanocrystals or nanotubes on the substrate.

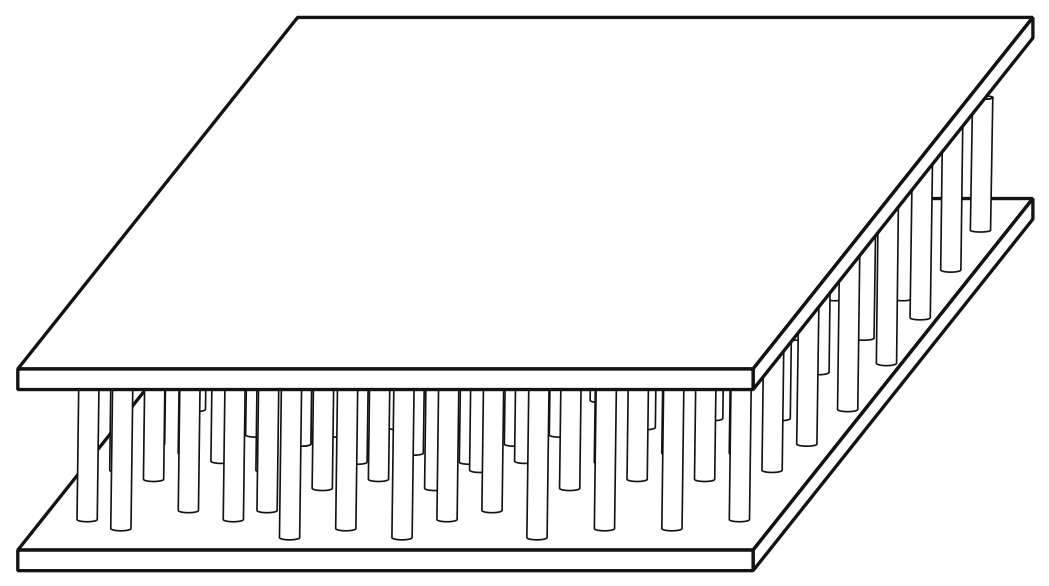

Fig. 3. Three-layer nanostructure formed by an array of nanocrystals or nanotubes placed between two plates.

\section{DYNAMICS OF THE SUBSTRATE-NANOOBJECTS SYSTEM}

1.1. Formulation of the Problem. Let us consider a system consisting of a horizontally aligned plate modeling the substrate and vertical rods modeling the nanoobjects (see Fig. 2). The plate occupies the domain $0 \leq x \leq a, 0 \leq y \leq b$; its mechanical properties are characterized by surface density $\rho$ and bending stiffness $D$. The rods occupy the domains $0 \leq z \leq l$; their mechanical properties are characterized by linear density $\rho_{*}$ and bending stiffness $C$. The lower ends of the vertically aligned rods are clamped on the plate and are located at identical distances from each other; therefore, their distribution density in the $x$ and $y$ directions is identical: $\zeta=M / a=N / b$ ( $M$ and $N$ are the numbers of rods in the $x$ and $y$ directions, respectively). The upper ends of the vertical rods are free, and the plate edges are simply supported. Note that the size of the plate modeling the substrate is much greater than the size of one nanoobject.

Bending oscillations of the nanoobjects are described by the equations of the classical theory of rods [16]

$$
C \boldsymbol{u}_{m n}^{\prime \prime \prime \prime}+\rho_{*} \ddot{\boldsymbol{u}}_{m n}=0,
$$

where $\boldsymbol{u}_{m n}$ is the vector of displacement of the rods in the horizontal direction with the corresponding ordinal numbers; the prime indicates the derivative with respect to the spatial coordinate $z$; the dot indicates the derivative with respect to time. The expressions for the remaining quantities characterizing the stress-strain state have the form

$$
\boldsymbol{\varphi}_{m n}=\boldsymbol{n} \times \boldsymbol{u}_{m n}^{\prime}, \quad \boldsymbol{L}_{m n}=C \boldsymbol{n} \times \boldsymbol{u}_{m n}^{\prime \prime}, \quad \boldsymbol{T}_{m n}=-C \boldsymbol{n} \times \boldsymbol{u}_{m n}^{\prime \prime \prime} .
$$


Here, $\boldsymbol{n}$ is the unit vector directed vertically upward, $\boldsymbol{\varphi}_{m n}$ is the vector of rotation of the rod sections, $\boldsymbol{L}_{m n}$ is the vector of the bending moments, and $\boldsymbol{T}_{m n}$ is the vector of shear forces. The rods are assumed to be inextensible; therefore, their vertical motion is described as

$$
v_{m n}^{\prime}=0, \quad F_{m n}^{\prime}=\rho_{*} \ddot{v}_{m n},
$$

where $v_{m n}$ are the displacements in the $z$ direction and $F_{m n}$ are the longitudinal forces.

The equations of motion of the plate have the form

$$
\begin{gathered}
\nabla \cdot \boldsymbol{N}+\left.\sum_{m=1}^{M} \sum_{n=1}^{N} F_{m n}\right|_{z=0} \delta\left(x-x_{m}\right) \delta\left(y-y_{n}\right)=\rho \ddot{w}, \\
\nabla \cdot M-\boldsymbol{n} \times \boldsymbol{N}+\left.\sum_{m=1}^{M} \sum_{n=1}^{N} \boldsymbol{L}_{m n}\right|_{z=0} \delta\left(x-x_{m}\right) \delta\left(y-y_{n}\right)=0 .
\end{gathered}
$$

Here, $w$ are the vertical displacements of the plate, $\boldsymbol{N}$ is the vector of transverse forces, $M$ is the tensor of moments, $\delta$ is the Dirac delta function, $\nabla \equiv \boldsymbol{e}_{1} \partial / \partial x+\boldsymbol{e}_{2} \partial / \partial y$ is the nabla operator in the plane $x, y$, and $\boldsymbol{e}_{1}$ and $\boldsymbol{e}_{2}$ are the Cartesian basis vectors orthogonal to $\boldsymbol{n}$. Below, we use the classical theory of plates [17], which implies the validity of the following relation between the rotation vector $\boldsymbol{\psi}$ and the transverse bending:

$$
\boldsymbol{\psi}=-\boldsymbol{n} \times \nabla w,
$$

while the tensor of moments is expressed in terms of the transverse bending by the formula

$$
M=D\left[\left(\frac{\partial^{2} w}{\partial y^{2}}+\nu \frac{\partial^{2} w}{\partial x^{2}}\right) \boldsymbol{e}_{1} \boldsymbol{e}_{2}-\left(\frac{\partial^{2} w}{\partial x^{2}}+\nu \frac{\partial^{2} w}{\partial y^{2}}\right) \boldsymbol{e}_{2} \boldsymbol{e}_{1}+(1-\nu) \frac{\partial^{2} w}{\partial x \partial y}\left(\boldsymbol{e}_{1} \boldsymbol{e}_{1}-\boldsymbol{e}_{2} \boldsymbol{e}_{2}\right)\right] .
$$

1.2. Natural Oscillations of the Nanostructure. Eliminating the vector of the transverse forces $\mathbf{N}$ from Eqs. (3) and using Eq. (5) for the tensor of moments, we convert system (3) to one scalar equation

$$
D \Delta \Delta w+\rho \ddot{w}=\sum_{m=1}^{M} \sum_{n=1}^{N}\left\{\left.F_{m n}\right|_{z=0} \delta\left(x-x_{m}\right) \delta\left(y-y_{n}\right)-\nabla \cdot\left[\boldsymbol{n} \times\left.\boldsymbol{L}_{m n}\right|_{z=0} \delta\left(x-x_{m}\right) \delta\left(y-y_{n}\right)\right]\right\} .
$$

Plate deformation in the plane $x, y$ is neglected. Then, the kinematic conditions of the junction between the rods and the plate can be written as

$$
\left.\boldsymbol{u}_{m n}\right|_{z=0}=0,\left.\quad v_{m n}\right|_{z=0}=\left.w\right|_{x=x_{m}, y=y_{n}},\left.\quad \boldsymbol{\varphi}_{m n}\right|_{z=0}=\left.\boldsymbol{\psi}\right|_{x=x_{m}, y=y_{n}} .
$$

The upper ends of the rods are free. The boundary conditions on these ends have the form

$$
\left.F_{m n}\right|_{z=l}=0,\left.\quad \boldsymbol{T}_{m n}\right|_{z=l}=0,\left.\quad \boldsymbol{L}_{m n}\right|_{z=l}=0
$$

Further we consider the problem of free oscillations of the system. We present the solution in the form

$$
\boldsymbol{u}_{m n}(z, t)=\boldsymbol{U}_{m n}(z) \mathrm{e}^{i \omega t}, \quad v_{m n}(z, t)=V_{m n}(z) \mathrm{e}^{i \omega t}, \quad w(x, y, t)=W(x, y) \mathrm{e}^{i \omega t},
$$

where $\omega$ is the eigenfrequency of system oscillations.

The study of the above-formulated problem shows that there are two groups of solutions. The first group of solutions corresponds to the case where the plate remains motionless, while the vertical rods move as cantilever beams. In this case, the eigenfrequencies $\omega$ of the system coincide with the eigenfrequencies of oscillations of the cantilever beams and are determined by solving the frequency equation

$$
1+\cos (\lambda l) \cosh (\lambda l)=0, \quad \lambda=\sqrt[4]{\rho_{*} / C} \sqrt{\omega} .
$$

Let us study the second group of solutions. Let the eigenfrequencies of the system be different from those that are solutions of Eq. (9). In this case, solving the equations of motion of the rods [Eqs. (1) and (2)] with the boundary conditions on the free ends (8), we can find the relation between the forces and displacements at the lower points of the rods

$$
\left.F_{m n}\right|_{z=0}=-\left.\rho_{*} l \ddot{v}_{m n}\right|_{z=0},\left.\quad \boldsymbol{L}_{m n}\right|_{z=0}=\left.\frac{C \lambda}{g(\lambda l)} \boldsymbol{\varphi}_{m n}\right|_{z=0}
$$



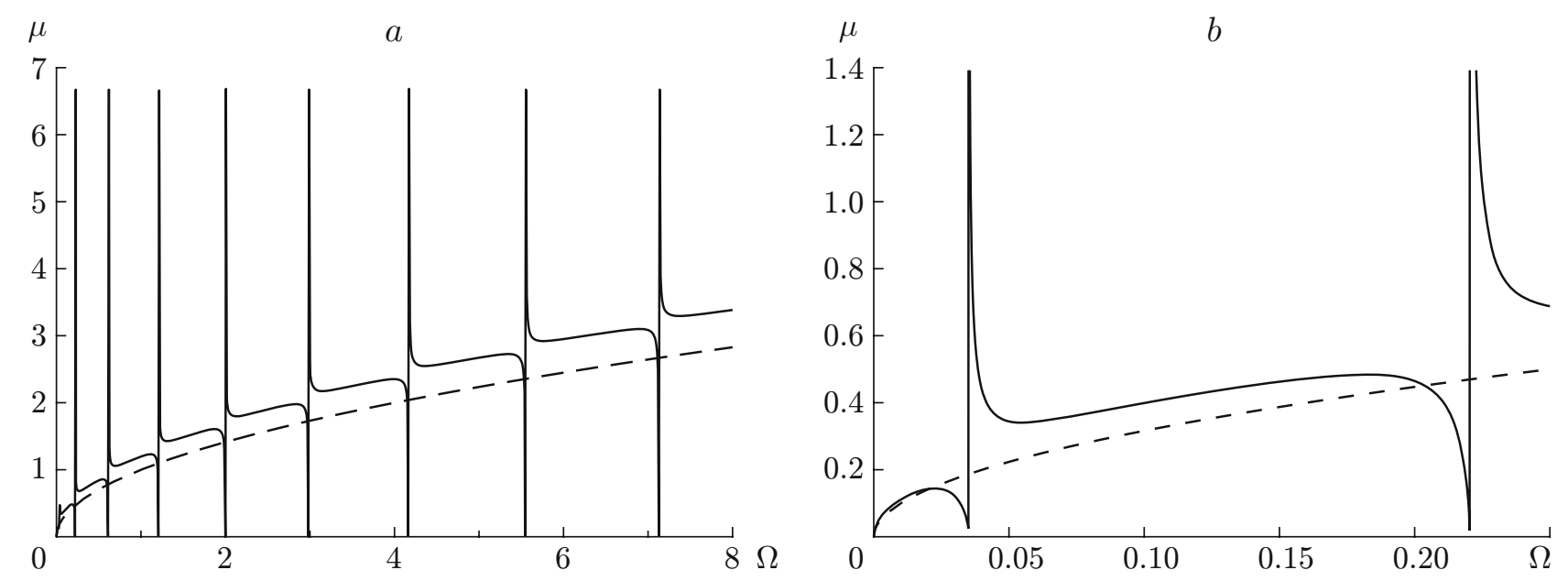

Fig. 4. Dispersion curves of the system (solid curves) and the substrate (dashed curves) obtained in different scales.

where

$$
g(\lambda l)=\frac{1+\cos (\lambda l) \cosh (\lambda l)}{\sin (\lambda l) \cosh (\lambda l)-\cos (\lambda l) \sinh (\lambda l)} .
$$

Note that the value of $g(\lambda l)$ vanishes at eigenfrequencies satisfying Eq. (9), and the relation between the vector of the bending moments and the rotation vector (10) becomes meaningless. Thus, the case with $g(\lambda l) \neq 0$ is further considered.

In view of Eqs. (4), (7), and (10), Eq. (6) is written in the form

$D \Delta \Delta W-\rho \omega^{2} W=\sum_{m=1}^{M} \sum_{n=1}^{N}\left(\rho_{*} l \omega^{2} W \delta\left(x-x_{m}\right) \delta\left(y-y_{n}\right)-\frac{C \lambda}{g(\lambda l)} \nabla \cdot\left[\left.(\nabla W)\right|_{x=x_{m}, y=y_{n}} \delta\left(x-x_{m}\right) \delta\left(y-y_{n}\right)\right]\right)$.

If there is a sufficiently large number of the vertical rods, they can be assumed to be continuously distributed over the plate area. Averaging the right side of Eq. (11), we simplify the mathematical formulation of the problem and reduce it to the equation

$$
D \Delta \Delta W-\zeta^{2} \frac{C \lambda}{g(\lambda l)} \Delta W-\rho \omega^{2}\left(1+\zeta^{2} \frac{\rho_{*} l}{\rho}\right) W=0,
$$

where $\zeta^{2}$ is the distribution density of the rods over the plate area (the distribution densities of the rods in the $x$ and $y$ directions are assumed to be identical). Presenting the solution of Eq. (12) in the form

$$
W(x, y)=W_{0} \exp \left(i\left[\mu_{1} x+\mu_{2} y\right] / l\right)
$$

we obtain the dispersion relation

$$
\mu^{4}+\frac{A \sqrt{\Omega}}{g(\sqrt{\Omega} / B)} \mu^{2}-(1+\xi) \Omega^{2}=0
$$

where

$$
\mu^{2}=\mu_{1}^{2}+\mu_{2}^{2}, \quad \Omega=\sqrt{\frac{\rho}{D}} l^{2} \omega, \quad A=\zeta^{2} \frac{C l}{B D}, \quad B=\left(\frac{\rho C}{\rho_{*} D}\right)^{1 / 4}, \quad \xi=\zeta^{2} \frac{\rho_{*} l}{\rho}
$$

( $\Omega$ is the dimensionless eigenfrequency, $\mu_{1}$ and $\mu_{2}$ are the dimensionless wavenumbers, and $A, B$, and $\xi$ are the dimensionless parameters with $A$ and $B$ being small quantities). The quantity $\xi$ can be either small or of the order of unity if, for instance, $l \sim h$ ( $h$ is the substrate thickness) and $\zeta h_{*} \sim 1$ ( $h_{*}$ is the characteristic diameter of the nanoobject cross section). Figure 4 shows the dispersion curves of the system and the substrate, which are plotted in different scales. The vertical portions of these curves correspond to the eigenfrequencies of the rods. 
Let us consider the solutions of the problem of free oscillations of the system. The solutions of the first group describe the free oscillations of the vertical rods on the motionless plate. As the plate is simply supported, the solutions of the second group have the form

$$
W_{m n}(x, y)=W_{m n} \sin (\pi m x / a) \sin (\pi n y / b)
$$

The corresponding frequency equation is written as

$$
(\pi l)^{4}\left[\left(\frac{m}{a}\right)^{2}+\left(\frac{n}{b}\right)^{2}\right]^{2}+\frac{(\pi l)^{2} A \sqrt{\Omega_{m n}}}{g\left(\sqrt{\Omega_{m n}} / B\right)}\left[\left(\frac{m}{a}\right)^{2}+\left(\frac{n}{b}\right)^{2}\right]-(1+\xi) \Omega_{m n}^{2}=0 .
$$

Here, $\Omega_{m n}$ are the eigenfrequencies of the system, which differ from the eigenfrequencies of oscillations of the rods $\Omega_{k}$ determines from the equation $g\left(\sqrt{\Omega_{k}} / B\right)=0$.

1.3. Forced Oscillations. Let us consider the problem of forced oscillations of the system under the action of external distributed loading, which can be presented as a series in the eigenforms of plate oscillations:

$$
Q(x, y, t)=\left[\sum_{m, n} Q_{m n} \sin \left(\frac{\pi m x}{a}\right) \sin \left(\frac{\pi n y}{b}\right)\right] \sin \left(\omega_{*} t\right)
$$

( $\omega_{*}$ is the driving force frequency). The problem solution has the form

$$
W(x, y, t)=\left[\sum_{m, n} W_{m n} \sin \left(\frac{\pi m x}{a}\right) \sin \left(\frac{\pi n y}{b}\right)\right] \sin \left(\omega_{*} t\right) .
$$

The coefficients $W_{m n}$ are calculated by the formula

$$
W_{m n}=Q_{m n}\left(l^{4} / D\right) /\left\{(\pi l)^{4}\left[\left(\frac{m}{a}\right)^{2}+\left(\frac{n}{b}\right)^{2}\right]^{2}+\frac{(\pi l)^{2} A \sqrt{\Omega_{*}}}{g\left(\sqrt{\Omega_{*}} / B\right)}\left[\left(\frac{m}{a}\right)^{2}+\left(\frac{n}{b}\right)^{2}\right]-(1+\xi) \Omega_{*}^{2}\right\}
$$

where $\Omega_{*}$ is the dimensionless frequency of the driving force. It can be easily seen that $W_{m n}$ turns to infinity if $\Omega_{*}=\Omega_{m n}$ [see Eq. (13)]. If $\Omega_{*}$ coincides with one of the eigenfrequencies of rod oscillations, then $W_{m n}$ vanishes:

$$
g\left(\sqrt{\Omega_{*}} / B\right)=0 \quad \Rightarrow \quad W_{m n}=0 .
$$

Thus, the plate oscillations are seen to decay at the eigenfrequencies of rod oscillations.

\section{DYNAMICS OF THE THREE-LAYER NANOSTRUCTURE}

The dispersion relations derived above are similar to the relations in the theory of shear-deformable plates. This analogy allows us to consider a three-layer nanostructure (see Fig. 3) within the framework of the Reissnertype theory of plates [18]. In contrast to the classical theory of plates [17], the above-mentioned theory takes into account the transverse shear strains, which are important factors in studying deformation of three-layer plates. It should be noted that the nanorods can interact with each other even if there is no matrix between them owing to the van der Waals or electrostatic forces. This fact allows us to consider the array of nanorods as an elastic layer possessing the effective properties of a transversely isotropic medium.

2.1. Constitutive Relations of the Theory of Shear-Deformable Plates. Let us consider a three-layer plate with a symmetric structure over its thickness (Fig. 5). The plate thickness is denoted by $h$, and the thicknesses of the inner layer and surface layers are indicated by $h_{c}$ and $h_{f}$, respectively. The plate behavior is described by the five-parameter theory of plates [19-21] with the displacements of the plate mid-surface $\boldsymbol{u}=u_{1} \boldsymbol{e}_{1}+u_{2} \boldsymbol{e}_{2}+w \boldsymbol{n}$ and the rotation vector $\boldsymbol{\psi}=\psi_{1} \boldsymbol{e}_{1}+\psi_{2} \boldsymbol{e}_{2}$ being considered as unknowns.

The equations of motion, the kinematic relations, and the equations of state of an isotropic plate symmetric over its thickness have the following form [19-21]:

$$
\begin{gathered}
\nabla \cdot T+\boldsymbol{q}=\rho \ddot{\boldsymbol{u}}, \quad \nabla \cdot M+\boldsymbol{T}_{\times}+\boldsymbol{m}=\rho \Theta \cdot \ddot{\boldsymbol{\psi}} ; \\
\varepsilon=\left((\nabla \boldsymbol{u}) \cdot a+a \cdot(\nabla \boldsymbol{u})^{\mathrm{t}}\right) / 2, \quad \gamma=\nabla \boldsymbol{u} \cdot \boldsymbol{n}+c \cdot \boldsymbol{\psi}, \quad x=\nabla \boldsymbol{\psi} ; \\
W(\varepsilon, \gamma, æ)=\frac{1}{2} \varepsilon \cdot \cdot A \cdot \varepsilon+\frac{1}{2} æ \cdot C \cdot x+\frac{1}{2} \gamma \cdot \Gamma \cdot \gamma ;
\end{gathered}
$$




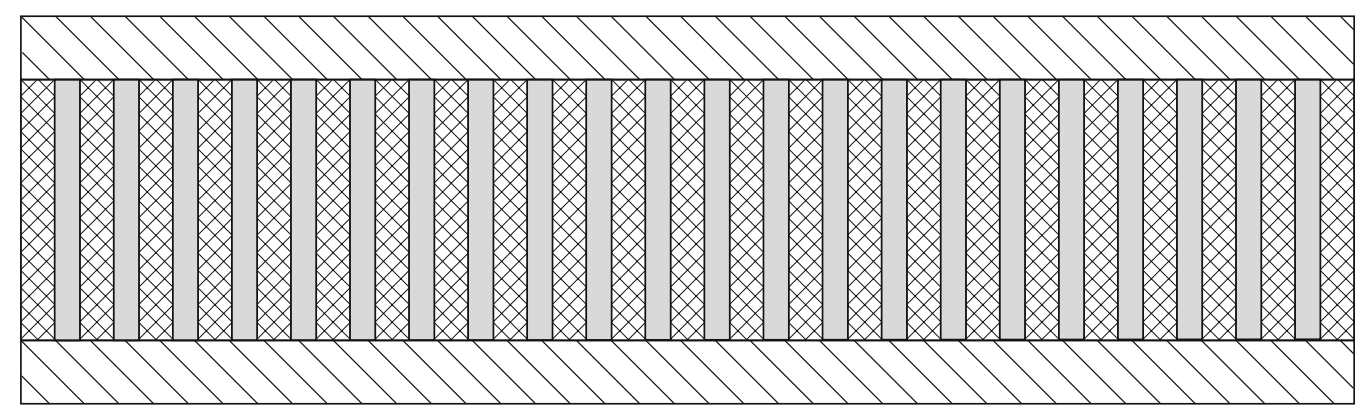

Fig. 5. Sketch of a three-layer plate with a nanocomposite inner layer.

$$
\begin{gathered}
T \cdot a=\frac{\partial W}{\partial \varepsilon}=A \cdot \varepsilon, \quad T \cdot \boldsymbol{n}=\frac{\partial W}{\partial \gamma}=\Gamma \cdot \gamma, \quad M^{\mathrm{t}}=\frac{\partial W}{\partial æ}=C \cdot \cdots \\
A=A_{11} a_{1} a_{1}+A_{22}\left(a_{2} a_{2}+a_{4} a_{4}\right), \quad C=C_{22}\left(a_{2} a_{2}+a_{4} a_{4}\right)+C_{33} a_{3} a_{3}, \\
\Gamma=\Gamma_{1} a_{1}+\Gamma_{2} a_{2}, \\
a_{1}=a=\boldsymbol{e}_{1} \boldsymbol{e}_{1}+\boldsymbol{e}_{2} \boldsymbol{e}_{2}, \quad a_{2}=\boldsymbol{e}_{1} \boldsymbol{e}_{1}-\boldsymbol{e}_{2} \boldsymbol{e}_{2}, \quad a_{3}=c=\boldsymbol{e}_{1} \boldsymbol{e}_{2}-\boldsymbol{e}_{2} \boldsymbol{e}_{1}, \quad a_{4}=\boldsymbol{e}_{1} \boldsymbol{e}_{2}+\boldsymbol{e}_{2} \boldsymbol{e}_{1} .
\end{gathered}
$$

In Eqs. (14)-(18), $T$ and $M$ are the tensors of forces and moments, $\boldsymbol{T}_{\times}$is the vector invariant of the tensor $T, \rho$ is the surface density of the plate, $\boldsymbol{u}$ is the displacement vector, $\boldsymbol{\psi}$ is the rotation vector, $\varepsilon$ is the tensile and shear strain tensor in the tangential plane, $æ$ is the bending and twisting strain tensor, $A, C$, and $\Gamma$ are the stiffness tensors of the shell, $a$ is the unit tensor in the tangential plane, $c=-a \times \boldsymbol{n}$, and $\Theta$ is the tensor of inertia; the superscript "t" means transposition.

The stiffness tensors $A, C$, and $\Gamma$ were determined in a number of papers (see [20-23]). For a plate made of an orthotropic material and having a symmetric structure over its thickness, the stiffness tensor components are determined by the formulas $[22,23]$

$$
\begin{gathered}
A_{11}=A_{22}=\frac{1}{4}\left\langle\frac{E_{1}+E_{2}+2 E_{1} \nu_{21}}{1-\nu_{12} \nu_{21}}\right\rangle, \quad A_{12}=\frac{1}{4}\left\langle\frac{E_{1}-E_{2}}{1-\nu_{12} \nu_{21}}\right\rangle, \\
A_{44}=\left\langle G_{12}\right\rangle, \quad C_{22}=\left\langle G_{12} z^{2}\right\rangle ; \\
C_{33}=C_{44}=\frac{1}{4}\left\langle\frac{E_{1}+E_{2}+2 E_{1} \nu_{21}}{1-\nu_{12} \nu_{21}} z^{2}\right\rangle, \quad C_{34}=-\frac{1}{4}\left\langle\frac{E_{1}-E_{2}}{1-\nu_{12} \nu_{21}} z^{2}\right\rangle, \\
\Gamma_{1}=\left(\lambda^{2}+\eta^{2}\right) C_{22} / 2, \quad \Gamma_{2}=\left(\eta^{2}-\lambda^{2}\right) C_{22} / 2,
\end{gathered}
$$

where $\langle(\cdot)\rangle=\int_{-h / 2}^{h / 2}(\cdot) d z$ is the operation of integration over the thickness, $\lambda^{2}$ and $\eta^{2}$ are the smallest eigenvalues of the boundary-value problems

$$
\begin{array}{ll}
\frac{d}{d z}\left(G_{2 n} \frac{d Z}{d z}\right)+\lambda^{2} G_{12} Z=0, & \left.\frac{d Z}{d z}\right|_{|z|=h / 2}=0, \\
\frac{d}{d z}\left(G_{1 n} \frac{d \tilde{Z}}{d z}\right)+\eta^{2} G_{12} \tilde{Z}=0, & \left.\frac{d \tilde{Z}}{d z}\right|_{|z|=h / 2}=0,
\end{array}
$$

$E_{1}, E_{2}, \nu_{12}, \nu_{21}, G_{12}, G_{1 n}$, and $G_{2 n}$ are the elastic moduli, which depend in the general case on the $z$ coordinate. For a transversely isotropic material, Eqs. (19) and (20) are substantially simplified because $E_{1}=E_{2}=E, \nu_{12}=$ $\nu_{21}=\nu, \nu_{n 1}=\nu_{n 2}=\nu_{3}, G_{12}=E /(2(1+\nu))$, and $G_{1 n}=G_{2 n}=G$. It follows from here that $A_{12}=C_{34}=\Gamma_{2}=0$, $A_{22}=A_{44}$, and $C_{22}=C_{44}$. 
The plate density and the tensor of inertia are described by the equations

$$
\rho=\left\langle\rho_{0}\right\rangle, \quad \rho \Theta=\Theta a, \quad \Theta=\left\langle\rho_{0} z^{2}\right\rangle,
$$

where $\rho_{0}(z)$ is the density of the three-dimensional body.

2.2. Analysis of Oscillations of a Three-Layer Plate. An analysis of the papers $[22,23]$ shows that it is possible to use the approximate relations derived in [24] for a three-layer plate with a symmetric structure over its thickness:

$$
D \equiv C_{22}+C_{33}=\frac{1}{4} \frac{E_{f} h^{2} h_{f}}{1-\nu_{f}^{2}}, \quad \Gamma=G_{c} h .
$$

Here, $E_{f}$ and $\nu_{f}$ are Young's modulus and Poisson's ratio for surface layers, and $G_{c}$ is the shear modulus for the inner layer (nanocomposite). It should be noted that $G_{c}$ substantially depends on the properties of the nanoobjects. Neglecting the matrix stiffness, we can demonstrate that

$$
G_{c}=\frac{12 N}{h_{c}^{2}} C,
$$

where $N$ is the number of nanoobjects per unit area and $C$ is the bending stiffness of one nanoobject. Thus, the stiffness $\Gamma$ depends on nanocomposite properties.

If the matrix density is ignored, then the values of $\rho$ and $\Theta$ can be determined by the relations

$$
\rho=\rho_{f} h_{f}+\frac{\pi \rho_{n} N d^{2}}{4} \rho_{n} h_{c}, \quad \Theta=\frac{\rho_{f}}{12}\left(h^{3}-h_{c}^{3}\right)+\frac{\pi \rho_{n} N d^{2}}{48} h_{c}^{3},
$$

where $\rho_{n}$ and $d$ are the density and diameter of the nanoobjects.

If there is no distributed moment $\boldsymbol{m}=0$, the equations of motion (14) can be converted to one equation [25]

$$
D \Delta \Delta w+\rho \ddot{w}+\frac{\rho \Theta}{\Gamma} w^{(4)}-\left(\Theta+\rho \frac{D}{\Gamma}\right) \Delta \ddot{w}=q_{n}+\frac{\Theta}{\Gamma} \ddot{q}_{n}-\frac{D}{\Gamma} \Delta q_{n},
$$

where $q_{n}=\boldsymbol{q} \cdot \boldsymbol{n}$ and $w^{(4)}=\partial^{4} w / \partial t^{4}$.

We seek for the solution of the homogeneous equation (23) in the form

$$
\bar{w}=W_{\mu} \exp (i \Omega \bar{t}) \exp \left(i \mu_{1} \bar{x}+i \mu_{2} \bar{y}\right),
$$

where

$$
\bar{w}=\frac{w}{h}, \quad \bar{x}=\frac{x}{h}, \quad \bar{y}=\frac{y}{h}, \quad \bar{t}=\frac{t}{T}, \quad T^{2}=\frac{\rho h^{4}}{D} .
$$

Nontrivial solutions of the homogeneous equation exist if the frequency $\Omega$ is related to the wavenumbers $\mu_{1}$ and $\mu_{2}$ by the dispersion relation

$$
L(\mu, \Omega) \equiv \mu^{4}-\Omega^{2}+\beta \Omega^{4}-\zeta \mu^{2} \Omega^{2}=0,
$$

where $\beta=\Theta D /\left(\rho \Gamma h^{4}\right)$ and $\zeta=(\Theta / \rho+D / \Gamma) / h^{2}$.

Typical dispersion curves are shown in Fig. 6. A comparison with the dispersion curves for a plate with nanoobjects (see Fig. 4) shows the absence of internal dynamics of nanoobjects, which cannot be taken into account within the framework of the Reissner-type theory of plates $[18,24]$. There are two branches of the dispersion curve indicated by $R_{1}$ and $R_{2}$. The high-frequency branch $R_{2}$ begins at the point $\left(0, \Omega^{*}\right)$, where the cutoff frequency $\Omega^{*}$ is determined by the formula

$$
\Omega^{*} \equiv \frac{1}{\sqrt{\beta}}=h^{2} \sqrt{\frac{\rho \Gamma}{\Theta D}} .
$$

In contrast to a homogeneous plate whose cutoff frequency is located in the high-frequency range, the cutoff frequency for the three-layer nanocomposite plate considered can vary in a wide range, including the regions of sufficiently low frequencies. Knowing the frequency $\Omega^{*}$, which can be found, for instance, from experimental data of studying the amplitude-frequency characteristic, and using Eqs. (21) and (22), we can estimate the bending stiffness of one nanoobject.

To find the amplitude-frequency characteristic, we consider forced oscillations of a rectangular plate with the sides of length $a$ and $b$, which is simply supported over its perimeter. Let the external load be determined by the formula

$$
\bar{q}=Q_{m n} \exp (i \Omega \bar{t}) \sin (\pi m h \bar{x} / a) \sin (\pi n h \bar{y} / b)
$$




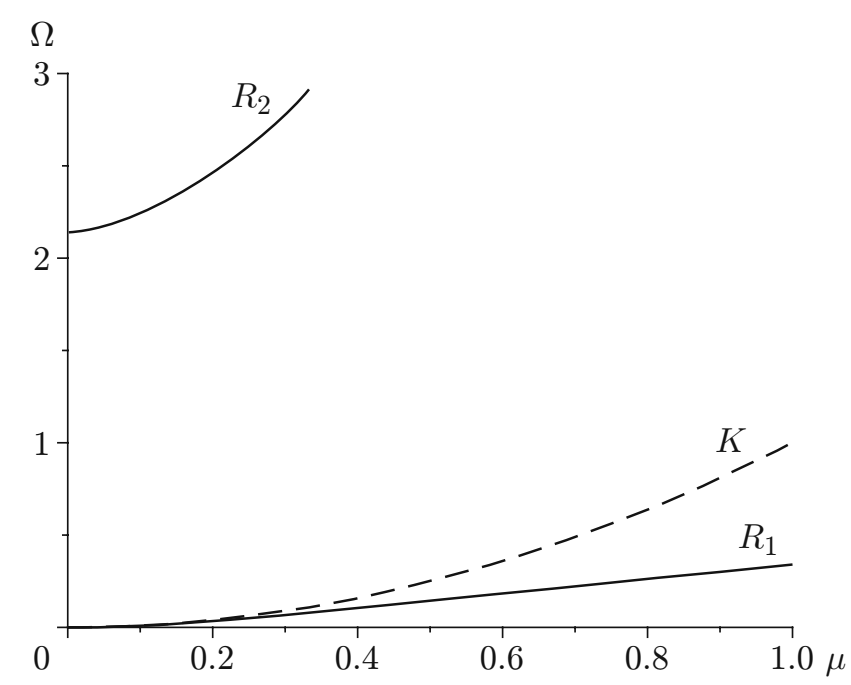

Fig. 6. Dispersion curves for a three-layer plate $(\Gamma=\infty$ and $\Theta=0): R_{1}$ and $R_{2}$ are the branches of the dispersion curve obtained with the Reissner theory; $K$ is the dispersion curve obtained by the Kirchhoff theory.

where $Q_{m n}=$ const. Then, the solution of Eq. (23) is written in the form

$$
\bar{w}=W_{m n} \exp (i \Omega \bar{t}) \sin (\pi m h \bar{x} / a) \sin (\pi n h \bar{y} / b),
$$

where the amplitude $W_{m n}$ is determined by the formula

$$
W_{m n}=\frac{1-\beta \Omega^{2}+\eta \mu^{2}}{L(\mu, \Omega)} Q_{m n}, \quad \mu^{2}=\frac{(\pi m h)^{2}}{a^{2}}+\frac{(\pi n h)^{2}}{b^{2}}, \quad \eta=\frac{D}{h^{2} \Gamma} .
$$

For rather smooth arbitrary loading, the solutions of Eq. (23) are presented as a sum of a series with terms of the form (24). Thus, the amplitude-frequency characteristic is completely determined by the amplitudes $W_{m n}$, which have singularities (poles) at eigenfrequencies.

\section{CONCLUSIONS}

Dispersion curves for some nanostructures formed by ordered arrays of nanoobjects (crystals or nanotubes parallel to each other) are constructed. The possibility of using these curves for determining elastic properties of nanoobjects from their resonance frequencies is discussed. The method of determining the properties of nanoobjects is based on experimental measurements of the spectrum of eigenfrequencies of a composite plate formed by a large number of almost identical nanorods grown on a substrate. Two possible nanostructures are considered: asymmetric (consisting of a substrate and an array of nanoobjects) and symmetric (three-layer plate whose inner layer is an array of nanoobjects). As is demonstrated above, the eigenfrequencies of such nanostructures are certain integral characteristics depending on the properties of nanoobjects. Thus, the eigenfrequencies depend on transverse shear stiffness, which, in turn, is expressed via the number of nanoobjects and their stiffness. In addition to the spectrum of composite nanostructures, it seems reasonable to determine the spectrum of similar homogeneous nanostructures, for instance, plates of the same geometry but made of the substrate material. A comparison of the nanostructure spectrum and the "reference" plate spectrum will allow identification of eigenfrequencies that exhibit the greatest dependence on the properties of nanoobjects.

As the dynamic characteristics of the nanostructures considered depend on the properties of nanoobjects, the results obtained can be used in the development of sensors that involve nanoarrays.

This work was supported by the Russian Foundation for Basic Research (Grant Nos. 06-01-00452 and 09-01-00459) and by the Council of the President of the Russian Federation for Supporting Young Russian Scientists (Grant No. MD-4829.2007.1) and Leading Scientific Schools (Grant No. NSh-2405.2008.1). 


\section{REFERENCES}

1. B. Bhushan (ed.), Springer Handbook of Nanotechnology, Springer-Verlag, Berlin (2007).

2. M. H. Huang, S. Mao, H. Feick, et al., "Room-temperature ultraviolet nanowire nanolasers," Science, 292, No. 5523, 1897-1899 (2001).

3. K. B. Lee, E. Y. Kim, C. A. Mirkin, and S. M. Wolinsky, "The use of nanoarrays for highly sensitive and selective detection of human immunodeficiency virus type 1 in plasma," Nano Lett., 4, No. 10, 1869-1872 (2004).

4. G. K. Mor, O. K. Varghese, M. Paulose, et al., "A review on highly ordered, vertically oriented $\mathrm{TiO}_{2}$ nanotube arrays: Fabrication, material properties, and solar energy applications," Solar Energy Mater. Solar Cells, 90, No. 14, 2011-2075 (2006).

5. V. K. Varadan and V. V. Varadan, "Microsensors, microelectromechanical systems (MEMS), and electronics for smart structures and systems," Smart Mater. Struct., 9, No. 6, 953-972 (2000).

6. L. Vayssieres, K. Keis, A. Hagfeldt, and S. E. Lindquist, "Three-dimensional array of highly oriented crystalline ZnO microtubes," Chem. Mater., 13, No. 12, 4395-4398 (2001).

7. M. Yu. Gutkin and I. A. Ovid'ko, Defects and Mechanisms of Plasticity in Nanostructural and Non-Crystalline Materials [in Russian], Yanus, St. Petersburg (2003).

8. M. Yu. Gutkin and I. A. Ovid'ko, Physical Mechanics of Deformable Nanostructures [in Russian], Vols. 1 and 2, Yanus, St. Petersburg (2003-2005).

9. A. M. Krivtsov and N. F. Morozov, "Anomalies of mechanical characteristics of nanosize objects," Dokl Ross. Akad. Nauk, 381, No. 3, 345-347 (2001).

10. W. A. Goddard, D. W. Brenner, S. E. Lyshevski, and G. J. Iafrate (eds.), Handbook of Nanoscience, Engineering, and Technology, CRC Press, Boca Raton (2003).

11. V. A. Eremeyev, E. A. Ivanova, N. F. Morozova, and A. N. Solov'ev, "Determining the eigenfrequencies of nanoobjects," Dokl. Ross. Akad. Nauk, 406, No. 6, 756-759 (2006).

12. V. A. Eremeyev, E. A. Ivanova, N. F. Morozova, and A. N. Solov'ev, "One method of determining the eigenfrequencies of an ordered system of nanoobjects," Zh. Tekh. Fiz., 77, No. 1, 3-8 (2007).

13. M. Lorenz, J. Lenzner, E. M. Kaidashev, et al., "Cathodoluminescence of selected single ZnO nanowires on sapphire," Ann. Physik, 13, Nos. 1/2, 39-42 (2004).

14. E. M. Kaidashev, M. Lorenz, H. Wenckstern, et al., "High electron mobility of epitaxial ZnO thin films on c-plane sapphire grown by multi-step pulsed laser deposition," Appl. Phys. Lett., 82, 3901-3903 (2003).

15. M. Lorenz, H. Hochmuth, R. Schmidt-Grund, et al., "Advances of pulsed laser deposition of ZnO thin films," Ann. Physik, Bd 13, Nos. 1/2, 59-61 (2004).

16. Yu. N. Rabotnov, Mechanics of a Deformable Solid [in Russian], Nauka, Moscow (1988).

17. S. P. Timoshenko and S. Woinowsky-Krieger, Theory of Plates and Shells, McGraw-Hill, New York (1959).

18. É. I. Grigolyuk and I. T. Selezov, Nonclassical Theories of Oscillations of Rods, Plates, and Shells [in Russian], VINITI, Moscow (1973). (Achievements of Science and Engineering, Ser. Mechanics of Deformable Solids, Vol. 5.)

19. P. A. Zhilin, "General equations of the non-classical shell theory," in: Tr. Leningrad. Polytekh. Inst., No. 386, 29-46 (1982).

20. H. Al'tenbach and P. A. Zhilin, "General theory of simple flexible shells," Usp. Mekh., No. 4, 107-148 (1988).

21. P. A. Zhilin, Applied Mechanics. Fundamentals of the Theory of Shells [in Russian], Izd. St.-Peterb. Univ., St. Petersburg (2006).

22. H. Altenbach, "An alternative determination of transverse shear stiffnesses for sandwich and laminated plates," Int. J. Solids Struct., 37, No. 25, 3503-3520 (2000).

23. H. Altenbach, "On the determination of transverse shear stiffnesses of orthotropic plates," Z. Angew. Math. Phys., 51, 629-649 (2000).

24. E. Reissner, "On bending of elastic plates," Quart. Appl. Math., 5, 55-68 (1947).

25. H. Altenbach and V. A. Eremeyev, "Eigen-vibrations of plates made of functionally graded material," Comput., Mater., Continua, 9, No. 2, 153-177 (2009). 